\title{
El enfoque de competencias en la formación de recursos humanos para bibliotecas
}

\author{
Lina Escalona Ríos * \\ Roberto Arriola Ruiz \\ Roselia A. Bautista **
}

Artículo recibido:

17 de enero de 2006.

Artículo aceptado:

18 de junio de 2008.

\section{RESUMEN}

El presente trabajo aborda el enfoque de competencias en la formación de recursos humanos para las bibliotecas, particularmente en los programas de capacitación que pueden aprovechar este enfoque en tanto que optimiza el desarrollo de las destrezas y competencias adecuadas para trabajar en una biblioteca. El enfoque de competencias se aplicó a la actualización de la Capacitación en Biblioteconomía del Colegio de Bachilleres de la Secretaría de Educación Pública, que tiene entre sus finalidades capacitar a los estudiantes para el trabajo, además de darles una formación propedéutica para continuar con estudios universitarios.

* Centro Universitario de Investigaciones Bibliotecológicas de la UNAM, México. escalona@cuib.unam.mx

** Los dos autores pertenecen al Colegio de Bachilleres, México.

INVESTIGACIÓN BIBLIOTECOLÓGICA, Vol. 22, Núm. 46, septiembre/diciembre, 2008, México, ISSN: 0187-358X. pp. 15-40 
Competencies focus in human resources education for libraries

Lina Escalona Ríos; Roberto Arriola Ruiz and Roselia A. Bautista

A competencies focus is discussed particularly with regard to program training to optimize the development of adequate skills and competency for library work. This focus was used to update training in Library Science word at SEP's (Public Education Ministry) Colegio de Bachilleres, whose purpose is to prepare students for the work place, besides giving them propaedeutic instruction to continue on with university studies.

Keywords: Competencies focus; Library Science training; Public education.

\section{INTRODUCCIÓN}

$\mathrm{D}$ esde hace algunas décadas (1970) el mundo está siendo transformado por el conocimiento aplicado al ámbito productivo que modifica la economía, el comercio, la política, la cultura, la educación y la forma de vida del ser humano. A esta época, en la que influye la generación de conocimiento, la información, la tecnología y las telecomunicaciones, se la conoce como Sociedad de la información o del conocimiento.

Los cambios impuestos por este tipo de sociedad requieren formar recursos humanos competitivos que puedan desempeñarse con calidad y responder así a los requerimientos sociales.

En la formación de recursos humanos el enfoque que está permeando la educación superior técnica y la capacitación es el enfoque de competencias, el cual integra competencias de conocimiento, de actitud y de procedimiento para lograr el desarrollo de habilidades que le permitan al capacitado ser una persona competitiva en el mercado actual de trabajo.

El área bibliotecológica en México forma recursos humanos para las bibliotecas y demás unidades de información en tres niveles educativos: capacitación técnica, profesional y de posgrado, el cual comprende la maestría y el doctorado. 
A nivel de capacitación técnica, la bibliotecología se les imparte como una opción a los estudiantes de nivel bachillerato en el Colegio de Ciencias y Humanidades de la Universidad Nacional Autónoma de México y en el Colegio de Bachilleres de la Secretaría de Educación Pública. El objetivo de este nivel es preparar a los estudiantes en un campo de trabajo específico para que se puedan desenvolver en éste al finalizar sus estudios, sin que dicha opción represente un obstáculo para continuar con sus estudios profesionales.

En el sector laboral bibliotecario la necesidad de personal auxiliar o técnico para desempeñar tareas bibliotecarias no profesionales es grande porque de acuerdo con las estadísticas de la Dirección General de Bibliotecas Públicas, sólo en este sector existen alrededor de 7100 instituciones; y a nivel nacional se cuenta con un total de 12, 836 bibliotecas de todo tipo (Instituto Nacional de Estadística, Geografía e Informática, 2003).

La estructura formal de empleo no ha cuantificado las necesidades de personal no profesional o técnico que requiere el país, con excepción de la prospectiva realizada en 1980, que calculaba que se llegaría al año 2000 con un déficit de 18,029 técnicos en biblioteconomía (Fernández, 1999). Lo anterior consideraba que habría una proporción de 4 técnicos o no profesionales por cada profesional. Por otra parte un cálculo flexible presupone la necesidad de que exista un bibliotecario profesional por cada 5000 habitantes. Estos datos intentan dar una idea de lo necesario que resulta capacitar al personal auxiliar de bibliotecas con un modelo educativo que sea eficaz para la sociedad actual.

Desde su creación, los programas de nivel técnico han tenido modelos curriculares que han respondido parcialmente a los requerimientos de formación laboral, por lo que al solicitarle al Colegio de Bachilleres la revisión del plan de estudios se hizo necesario investigar, analizar y elegir un modelo educativo que fuese conveniente para lograr el desarrollo de las habilidades y competencias necesarias para que el egresado de este nivel pudiese trabajar en bibliotecas.

Al hacer el diagnóstico del plan de estudios se encontró que el perfil del egresado estaba orientado fundamentalmente hacia la realización de actividades propias del perfil de formación que un profesional de la biblioteconomía debía realizar y no hacia el desarrollo de las actividades de apoyo que un auxiliar y/o capacitado podría llevar a cabo en las unidades de información.

Por otra parte, el nombre de la capacitación así como algunas de sus asignaturas son similares a las impartidas en el nivel profesional, lo que genera falsas expectativas en los estudiantes, especialmente cuando los contenidos y las lecturas corresponden a ese nivel.

Dado lo anterior se hacía imprescindible reestructurar el plan y se seleccionó el modelo que considera el enfoque de competencias para lograr un plan integral acorde con los requerimientos actuales de la sociedad. 


\section{a) Enfoque de competencia laboral en el contexto educativo}

El enfoque de competencia laboral en el ámbito educativo no es nada nuevo porque ya Platón (Thurow, 1978) hacía alusión a los beneficios que la sociedad tendría al contar con personal bien entrenado para desempeñar alguna actividad productiva. Después, en 1776, Smith (Thurow, 1978) mencionaba que la diferencia entre los salarios de un trabajador adiestrado y los de un trabajador común se basaban en el principio del aprendizaje, por lo que el trabajador adiestrado ganaba más porque, de acuerdo con lo aprendido, producía más.

Pero es Theodore Schultz (2000) quien relaciona el concepto de capital con el del hombre y la educación que éste recibe, y concibe la teoría del Capital Humano. Este autor defiende la idea de que el hombre invierte recursos (tiempo, dinero, etcétera), en educarse o capacitarse para desempeñarse en alguna actividad y que una vez capacitado espera la retribución por lo que hace, ya que la producción de lo realizado es de mayor calidad; es decir, la capacitación lo ha convertido en un hombre competente para realizar la actividad encomendada.

El proceso de globalización nos lleva a retomar la teoría de Schultz y el papel que juega la educación como formadora de personas que el mercado global está requiriendo, por lo que surge la educación basada en competencias, la cual ha sido apoyada y promovida por organismos internacionales que marcan las directrices y políticas educativas. Así, la Conferencia Mundial sobre la Educación, celebrada en 1998 en la sede de la UNESCO, expresó que es necesario propiciar el aprendizaje permanente y la construcción de las competencias adecuadas para contribuir al desarrollo cultural, social y económico de la Sociedad de la Información (Argudin, 2005).

Pero en términos generales ¿qué es una competencia?

De acuerdo con las raíces latinas: cum y petere, competencia es "la capacidad para concurrir, coincidir en la dirección” y de acuerdo con Argudin (2005) significa seguir el paso o seguir en un área determinada.

Por su parte la UNESCO la define en 1999, como:

El conjunto socioafectivo y las habilidades cognoscitivas, psicológicas, sensoriales y motoras que permiten llevar a cabo adecuadamente un desempeño, una función, una actividad o una tarea (Argudin, 2005).

De tal forma, las competencias son las habilidades desarrolladas que se tienen para realizar una determinada actividad con buena calidad.

El concepto de competencia vinculado a actividades laborales se usa a partir de los resultados obtenidos por los trabajos de investigación de David 
McClelland (1994) en los años setenta, cuando se pretendía identificar las variables que explicaran el desempeño laboral, para lo cual se utilizaron las técnicas de entrevista y observación. La investigación analizó las peculiaridades de las actividades y el comportamiento del personal; es decir el proceso que éste llevaba a cabo vinculándolo con la productividad. Así, de acuerdo con la relación competencia/mercado de trabajo, la educación tendría que formar a las personas para que se insertaran en dicho mercado con las competencias adecuadas para hacer eficiente la productividad que lleve al desarrollo social y económico de cualquier comunidad o país.

De esta forma se vinculan las competencias del mercado laboral con la educación y desde esta perspectiva, la ANUIES define a la educación basada en competencias, como aquella que

[...]se fundamenta en un currículo apoyado en las competencias de manera integral y en la resolución de problemas. Utiliza recursos que simulan la vida real: análisis y resolución de problemas, que aborda de manera integral; trabajo cooperativo o por equipos, favorecido por tutorías (Argudin, 2005).

En el Espacio Europeo de Educación superior se define el término competencia como una

[...] combinación dinámica de atributos — respecto del conocimiento y sus aplicaciones, de las actitudes y responsabilidades — que describe los objetivos de aprendizaje de un programa educativo, o cómo los estudiantes habrán de ser capaces de actuar al final de dicho proceso (Escalona p. 15, cita a González, J y Wagenaar, Reds).

De acuerdo con estas dos concepciones el enfoque de competencias, aplicado al ámbito educativo, conlleva un proceso que integra a todos los elementos que intervienen en un proceso educativo y relaciona el modelo educativo con el propósito del programa, con la malla curricular, con las estrategias de aprendizaje y las de enseñanza y con la forma de evaluar. Es necesario visualizar el plan de estudios a partir de las competencias que se quieren desarrollar en los estudiantes, de tal forma que se transformen los objetivos de aprendizaje en competencias que hay que desarrollar y los exámenes "tradicionales" de evaluación de conocimiento se transformen en evidencias de conocimiento del producto o del desempeño que tendrán que ser vigilados por el docente como coordinador y facilitador del desarrollo de la competencia.

En ese sentido el papel del maestro cambia radicalmente y de ser el responsable del proceso enseñanza-aprendizaje se convierte en un docente que 
coordina las diversas actividades que llevan a cada alumno a desarrollar sus propias habilidades, que tienen como base su propio conocimiento.

Dado lo anterior, también el papel del alumno cambia de forma drástica, pues el enfoque de competencias tiene como base la participación activa del estudiante ya que se convierte en el fin y el centro de aprendizaje, por lo que es necesario reforzar el desarrollo del pensamiento crítico del estudiante, con objeto de que éste cuente con herramientas que le permitan discernir, deliberar y elegir libremente, de tal forma que pueda comprometerse con la construcción de sus propias competencias. De esta forma se garantiza que la educación basada en competencias le permita al educando crecer en sus dimensiones de persona, dándole coherencia a las necesidades de la sociedad y a su propio proyecto de vida. Asimismo, cuando el estudiante consolide su compromiso social, podrá reflexionar y actuar para que las competencias que construya sean el resultado de su desarrollo como persona para su sociedad (cfr. Argudin, 2005).

Bajo este enfoque la culminación de un nivel educativo no significa la terminación de la preparación, en el enfoque por competencias se prepara al alumno para ser un ente en continua formación en donde las competencias se expresan a través de la práctica de cada día a lo largo de toda la vida en un constante y renovado aprendizaje.

Cabe señalar que aunque el enfoque de competencias nos lleve necesariamente a un "saber hacer", este conocimiento práctico que debe demostrar el estudiante tiene como base un conocimiento teórico necesario para entender la competencia desarrollada y contextualizarla para ser aplicada en la resolución de problemas de forma apropiada. Es decir, no se trata de practicar un oficio o repetir continuamente una actividad hasta dominarla, sino fomentar la adquisición de conocimiento para comprender y realizar de forma efectiva esa actividad. Todo ello enmarcado en un conjunto de valores que tendrán que fomentarse en el espacio del aprendizaje.

De acuerdo con lo anterior, la elección del modelo educativo por competencias implica que el plan de estudios contemple las siguientes competencias:
a) Competencias cognoscitivas
b) Competencias de desempeño
c) Competencias de producto
d) Competencias actitudinales

Las competencias cognoscitivas proporcionarán el conocimiento teórico que fundamente las competencias de desempeño y producto. La redacción 
de dicha competencia comprenderá el qué se va a aprender, el cómo se va a lograr dicho aprendizaje y el para qué se va a aprender, para lograr así la integración de la competencia cognoscitiva.

Las competencias de desempeño son aquellas que demuestran el proceso aprendido, la forma en que se realiza la actividad y en que es evaluada bajo la observación de la forma y el razonamiento que el estudiante sigue en su realización.

Las competencias de producto son la evidencia de que el alumno ha logrado concretar las competencias congnoscitivas y de desempeño en una obra determinada, bajo estándares de calidad, que entonces pueden ser evaluados.

Para lograr un plan de estudios integral deben considerarse también las competencias actitudinales que implican tanto las actitudes como los valores que se deben tener y fomentar para el desarrollo efectivo de una actividad determinada.

\section{b) El enfoque de competencias aplicado al plan de capacitación en biblioteconomía}

Cualquier enfoque, corriente o modelo educativo debe tener congruencia con los objetivos institucionales, en este caso el Colegio de Bachilleres, a través de su plan de estudios, pretende brindar una formación propedéutica general a fin de que el estudiante acceda a estudios superiores con una cultura básica amplia y como parte de ésta recupera a la capacitación para el trabajo como un contenido curricular y al "trabajo" como una categoría de formación, en su sentido filosófico y educativo, con lo cual acrecienta los conocimientos y posibilidades de ingreso al mercado de trabajo de los bachilleres.

Así, el Colegio se plantea los siguientes como objetivos generales:

a) Desarrollar la capacidad del estudiante, mediante la obtención y aplicación de conocimientos.

b) Concederle la misma importancia a la enseñanza que al aprendizaje.

c) Crear en el estudiante una conciencia crítica que le permita adoptar una actitud responsable ante la sociedad.

d) Proporcionarle al estudiante capacitación y adiestramiento en una técnica o especialidad determinada, (Decreto de creación y estatuto general, 1975).

En este mismo sentido, el artículo $1^{\circ}$ del Acuerdo 71 de la SEP (1982), establece que el bachillerato es: 
... un ciclo de estudios que tiene como antecedente la educación secundaria. Su finalidad esencial es generar en el educando el desarrollo de una primera síntesis personal y social que le permita su acceso a la educación superior, a la vez que le dé una comprensión de su sociedad y de su tiempo y lo prepare para su posible incorporación al trabajo productivo (Modelo, 1998).

El Modelo Educativo, como documento rector en el que se explicitan los lineamientos de tipo jurídico, filosófico, psicopedagógico y curricular que orientan la organización y desarrollo de la práctica educativa, además del perfil de egreso del bachiller al finalizar la capacitación para el trabajo, el cual consiste en:

- Comprender el carácter histórico y cultural del trabajo y reconocer a éste como factor del desarrollo personal, social y económico.

- Aplicar los principios, leyes y conceptos básicos de los procesos de trabajo de un campo específico.

- Conocer las normas jurídicas propias del ámbito laboral de su campo de trabajo.

- Conocer las características principales de diferentes formas de organización para el trabajo.

- Manejar los instrumentos, herramientas, técnicas y procedimientos inherentes a un campo de trabajo.

- Realizar los procesos de trabajo relativos a un campo de trabajo.

- Resolver problemas propios de un campo laboral.

- Manifestar iniciativa y creatividad en la realización y en la solución de problemas inherentes a su trabajo.

- Mostrar que se comprende el sentido de responsabilidad social y económica que implica la realización de un trabajo.

- Estar dispuesto a comprender y aplicar los avances científicos y tecnológicos en la realización y mejoramiento del trabajo.

Con objeto de llevar a cabo los objetivos, finalidad y perfil del egresado, el Colegio estructura su plan de estudios en Áreas de Formación: básica, específica, para el trabajo y cultural, artística y deportiva (opcional).

De manera particular, el Área de Formación para el Trabajo tiene como finalidad:

Ofrecer al estudiante los elementos conceptuales y metodológicos de un conjunto de disciplinas que le permitan fortalecer sus conocimientos, habilidades cognitivas, valores y actitudes, para desarrollar procesos de trabajo de un campo laboral 
específico, así como reconocer el valor social y las responsabilidades que éste implica (Modelo Educativo, 1998, p. 44).

Basándose en este marco institucional, el Colegio de Bachilleres renueva su misión, orientada a:

Formar ciudadanos con un proyecto de vida basado en "Competencias Académicas y Laborales” y vocación profesional, con alta autoestima y compromiso consigo mismos, con su familia y la sociedad; mediante procesos educativos eficientes, que con libertad y calidad propicien su inventiva, retención, comprensión, creatividad y crítica; forjando hábitos de trabajo y principios éticos que normen su conducta para incorporarse productivamente a la sociedad y puedan continuar estudiando (Agenda, 2002).

Al respecto cabe señalar que el enfoque de competencia laboral que orienta la formación de los bachilleres en el ámbito de la formación para el trabajo, se recupera en la capacitación de biblioteconomía, a partir de que:

- Orienta la formación hacia la demanda de las necesidades sociales y laborales del área. Se diseña en función a la demanda y a las necesidades productivas y sociales que el país requiere.

- Amplía las posibilidades de que alumnos capacitados puedan emplearse. En la medida que su formación responde a demandas y requerimientos actuales de las Unidades de Información, aumentan sus posibilidades para integrarse al sector productivo.

- Genera la articulación entre la formación que se recibe en la institución y el sector productivo. Al impulsar la realización de prácticas laborales en diversas Unidades de Información, el diseño de situaciones lo más cercanas al ámbito laboral, así como al reconocer las competencias que se requieren para dar cuenta de la función productiva que se debe aprender durante la capacitación.

- Integra el "saber hacer", el "saber" y el "saber ser". La integración del conocimiento en los estudiantes se produce al ser éstos capaces de efectuar las funciones productivas que se plantean en el diseño curricular de la capacitación, tomando en cuenta los requerimientos de calidad para realizar un desempeño eficaz.

- Impulsa la capacidad productiva y competitiva de los estudiantes capacitados. Se acrecienta de manera significativa su capital humano, al tener un desempeño de calidad y eficiencia que satisfaga las necesidades y resuelvan las situaciones problemáticas a las que se enfrentan en su 
actividad cotidiana en las Unidades de Información, pues se reconocen como útiles e importantes en la función que llevan a cabo. En este aspecto, el proceso de normalización de diversas funciones productivas, cumple un papel muy importante como referente para el diseño de los programas y la evaluación de los resultados obtenidos al término del proceso de formación.

- Reconoce el proceso educativo como permanente, para toda la vida. Capacitarse para aprender, y aprender cada día más. La capacitación en biblioteconomía genera en sus estudiantes un conjunto de competencias que les serán útiles para los distintos ámbitos de su vida: escolar, laboral, familiar, etcétera.; competencias que son el inicio y el reconocimiento de un saber sobre el campo laboral que nunca termina.

- Enriquece los procesos de evaluación en cuanto a la formación para el trabajo. Reconociendo lo que los alumnos son capaces de "saber", "saber hacer" y "saber ser" en el contexto y las situaciones determinadas de las unidades de información.

- Flexibiliza la organización curricular. La generación del diseño curricular por módulos de aprendizaje flexibiliza la organización de los contenidos de aprendizaje y el tránsito entre éstos.

El enfoque de competencia laboral, sugerido por la UNESCO para la educación técnica, impacta en los procesos de enseñanza, aprendizaje y evaluación, y si bien no implica cambios o posturas excluyentes con el enfoque constructivista, el cual da sustento al modelo educativo del Colegio, sí requiere la explicitación de los lineamientos metodológicos que deben ser considerados para llevar a cabo el proceso de formación de competencias laborales.

La pregunta clave consiste en ¿cómo los estudiantes construyen las competencias laborales, cómo las aprenden? cuestionamiento que obliga a definir el papel del profesor y el del alumno, los medios y los métodos de aprendizaje, y también el proceso de evaluación del aprendizaje.

Los resultados de aprendizaje que ahora se esperan en la Capacitación en Servicios Bibliotecarios y de Información deben responder a competencias laborales que impliquen una estructuración lógica de conceptos, habilidades y actitudes que permitan el desarrollo de una función productiva con un sentido histórico social. En este sentido, la formación en competencia laboral requerirá de un proceso de integración que se efectúe desde el "saber hacer", pero que no se reduzca a éste.

Teniendo como base los niveles que plantean Acevedo y García (2000) para la construcción de competencias, y que consisten en: reconocimiento y 
distinción de elementos conceptuales, procedimentales y actitudinales; interpretación y uso de elementos conceptuales, procedimentales y actitudinales; y producción y generalización; las competencias a desarrollar en la capacitación requieren de la integración lógica de:

- La comprensión de los elementos conceptuales, procedimentales y actitudinales que posibiliten la realización de la competencia para organizar, registrar y conservar el acervo que proporcionan los servicios bibliotecarios.

- La organización, integración y aplicación de dichos elementos a través de prácticas escolares y de campo.

- La formulación y resolución de problemas del campo laboral que posibiliten la transferencia y generalización de las competencias laborales a otros contextos como el escolar.

- La explicitación del marco contextual que le otorgue sentido a las competencias que desarrollará en la Capacitación y que le permitirá reconocer el valor social que cobra la formación en esta área, en tanto que se vive en la llamada "sociedad de la información, del conocimiento".

En este sentido es importante advertir que el riesgo de generar una "simple instrumentalización", mecanización, de conceptos y procedimientos aislados de contexto alguno, como lo expresan Cárdenas y Sarmiento (2000):

...La simple resolución mecánica de ejercicios no es suficiente para el desarrollo de esta competencia; es preciso exponer a los estudiantes a condiciones en las cuales ejerciten la mente en la interpretación de situaciones problemáticas, permitirles que propongan y desarrollen sus propias iniciativas de solución, las analicen y determinen si las respuestas obtenidas son o no las más adecuadas; solamente de esta manera se aprende - incluso a partir de los propios errores - y se desarrolla la capacidad analítica e interpretativa que caracteriza a quienes analizan y solucionan situaciones problemáticas...

En todo caso se espera que el proceso de formación en competencias permita, como lo expresa Shön (citado por Ríos), "[...]pensar en lo que se hace mientras se está haciendo", rebasando el modelo reproduccionista del conocimiento. Al respecto, Gonczi incorpora el concepto de aprendizaje como desarrollo del pensamiento práctico, que consiste en: combinar una base de conocimientos específicos con las exigencias prácticas, articulando el conocimiento de procedimientos y proposiciones acompañadas de un control significativo en la resolución de problemas reales de trabajo (Gonczi , 1997). 
Con este marco se fortalece la necesidad de impulsar la concepción del profesor como mediador entre las competencias que han que desarrollar, y el estudiante como generador de situaciones de enseñanza y aprendizaje que contribuyan a:

- Organizar y presentar lógicamente la competencia de aprender ("saber" y "saber ser" a través del "saber hacer").

- Vincular los procesos de formación escolar con situaciones reales de trabajo de las Unidades de Información.

- Generar prácticas escolares que posibiliten la construcción de las competencias a través de situaciones problema del campo relacionado con los servicios bibliotecarios y de información.

- Mostrar y demostrar las ventajas de la formación por competencia laboral para realizar un servicio bibliotecario de calidad.

- Generar situaciones de aprendizaje que posibiliten la reflexión, el análisis y la síntesis de lo aprendido, considerando los errores, aciertos y experiencias como elementos del proceso de formación.

- Establecer momentos para demostrar y elaborar evidencias de aprendizaje de las competencias planteadas, como información sustancial que reoriente y fortalezca los procesos de enseñanza y aprendizaje.

Como se observa, ya no es suficiente con un profesor que repita información a partir de temas preestablecidos, ahora se requiere de un docente capaz de generar situaciones de aprendizaje que posibiliten la construcción de competencias laborales en los bachilleres y los haga actuar de manera responsable, eficaz y consciente.

Desde este marco se concibe al alumno como un sujeto:

- Activo y que cuenta con conocimientos previos acerca de la información, las bibliotecas, los documentos y los servicios bibliotecarios como conceptos clave para desarrollar las competencias laborales en la capacitación.

- Responsable de su propio proceso de aprendizaje, en tanto que nadie puede aprender por él, y no como un simple reproductor de tareas mecanizadas.

- Capaz de hacer uso de sus recursos cognitivos, afectivos-sociales y psicomotrices para aplicar los conocimientos aprendidos, explicando qué hace para organizar los recursos de información, sistematizar y recuperar información, proporcionar servicios a usuarios y conservar los documentos; así como, para qué realiza tal función productiva en una Unidad de Información 
- Capaz de reconocer el valor social de su desempeño como estudiante y como posible trabajador de una Biblioteca o Unidad de Información.

- Competente para resolver problemas haciendo uso de los conocimientos aprendidos, su experiencia laboral y su ingenio y creatividad.

En cuanto a los métodos de enseñanza y aprendizaje, tanto el enfoque en competencias como el constructivista reconocen el valor que cobra la formación a través de problemas. Particularmente en cuanto al desarrollo de competencias, Gonczi (1997) señala que las características de estos métodos son:

- Abordar problemas reales del campo laboral.

- Utilizar materiales que simulan la vida real.

- Disponer de recursos que les permitan a los educandos analizar los problemas y enfrentarlos.

- Trabajar cooperativamente entre los educandos y tener acceso a un tutor.

- Tratar un problema a la vez, en comparación con los cursos basados en materias, donde se busca abarcar muchos temas al mismo tiempo.

Con base en este marco, el desarrollo de competencias en la capacitación recupera como herramienta metodológica la formulación de problemas del campo laboral de las Bibliotecas y Unidades de Información como espacios de reflexión, construcción y aplicación de las competencias que hay que desarrollar.

La evaluación forma parte de los procesos de enseñanza y aprendizaje dentro del enfoque de competencias, y cobra especial valor por el impacto y la toma de decisiones que implica la información recabada en este proceso.

En el Colegio de Bachilleres la evaluación del aprendizaje se ha entendido como

...un proceso cuyo propósito es obtener información capaz de regular el proceso de enseñanza y aprendizaje, para ajustarlo a una mayor eficiencia; así como determinar si los aprendizajes establecidos se han logrado, para fundamentar la toma de decisiones sobre la acreditación del estudiante (Orientaciones, 1997).

Esta concepción de evaluación del aprendizaje se reconoce en el Colegio como un proceso continuo, sistemático y consciente, cuyos propósitos son:

...obtener información capaz de regular las estrategias de enseñanza y las experiencias de aprendizaje;

fundamentar la toma de decisiones sobre la acreditación del estudiante, y

fomentar en el alumno una actitud de responsabilidad ante sus resultados de 
aprendizaje.

Lo anterior, confiere a la evaluación del aprendizaje dos funciones: reguladora y decisoria.

La función reguladora permite al profesor orientar, valorar y ajustar su estrategia didáctica y los materiales empleados, así como realimentar al estudiante en cuanto a sus alcances y limitaciones en el logro de los objetivos.

La función decisoria, permite al profesor dictaminar sobre la acreditación del curso para cada alumno (Orientaciones, 1997).

En congruencia con el Modelo Educativo del Colegio, el proceso de evaluación del proceso de aprendizaje se conforma por tres tipos de evaluación: diagnóstica, formativa y sumativa, que vinculándolos con lo que señala el Consejo de Normalización y Certificación de Competencia Laboral (CONOCER), se convierte en:

...el procedimiento mediante el cual se recogen suficientes evidencias sobre el desempeño laboral de un individuo, de conformidad con una NTCL (Norma Técnica de Competencia Laboral). Lo anterior significa que las NTCL constituyen los referentes fundamentales de las evaluaciones y de su consecuencia, la certificación. (Qué es la evaluación...)

Entre las características que se consideran en la evaluación de competencia laboral están las siguientes:

- Se centran en los resultados del desempeño laboral (definidos en la norma).

- Están centradas en el aprendiz.

- Son de tipo individual.

- Tienen un carácter holístico, en tanto que se consideran como un elemento necesario en los procesos de desarrollo de la competencia.

- Son realizadas por personal que no está necesariamente involucrado en el proceso de formación.

- No establecen un tiempo determinado para su realización.

- No están asociadas a un curso o programa de estudio.

- No utilizan escalas de puntuación.

- Se realizan preferentemente en el campo de trabajo real, o en su caso se simula ésta.

En este planteamiento la evaluación permite, a través de las evidencias recopiladas y mostradas, valorar si el evaluado es competente o no para realizar la función productiva en la que se ha formado. 
Desde este marco, y en congruencia con el Modelo Educativo del Colegio y el enfoque de competencia laboral, la evaluación del aprendizaje en la Capacitación en Servicios Bibliotecarios y de Información, presenta las siguientes características:

- La evaluación comprende tres tipos: diagnóstica, formativa y sumativa.

- Tiene dos funciones centrales: reguladora, de los procesos de aprendizaje y enseñanza, y decisoria en cuanto a la acreditación del alumno.

- Se basa en la recopilación de evidencias que den cuenta de la competencia desarrollada (concentradas en el portafolio de evidencias).

- Está centrada en el aprendiz.

- Tiene un carácter holístico, en tanto que se considera como un elemento necesario en los procesos de desarrollo de la competencia.

- Se define claramente qué y cómo se evaluará el módulo de aprendizaje.

- Se realiza a través de simuladores lo más cercanos posibles al ambiente laboral.

A partir de este marco explicativo las diferentes modalidades de evaluación permiten recopilar información en cuanto a:

- ¿Para qué evaluar?

- ¿Qué evaluar?

- ¿Cuándo evaluar?

Como se muestra en la siguiente tabla: 
Tabla No. 1. La evaluación del aprendizaje

\begin{tabular}{|c|c|c|}
\hline \multicolumn{3}{|c|}{ ¿Para qué evaluar? } \\
\hline Diagnóstica & Formativa & Sumativa \\
\hline $\begin{array}{l}\text { - Obtener información sobre el } \\
\text { estado inicial que los estudian- } \\
\text { tes tienen de los conocimientos } \\
\text { previos en relación con las com- } \\
\text { petencias propuestos para cada } \\
\text { módulo, como necesarios para } \\
\text { lograr un desempeño efectivo; } \\
\text { con objeto de orientar el proceso } \\
\text { de formación. } \\
\text { - Identificar si los estudiantes ya } \\
\text { cuentan con los conocimientos } \\
\text { para desarrollar la competen- } \\
\text { cia propuesta en el módulo de } \\
\text { aprendizaje, con el fin de orien- } \\
\text { tar al profesor y estudiantes en } \\
\text { cuanto a una valoración global } \\
\text { del módulo para suacreditación. }\end{array}$ & $\begin{array}{l}\text { - Reconocer los avances y proble- } \\
\text { mas en el proceso de formación } \\
\text { de la competencia planteada en } \\
\text { el módulo de aprendizaje. } \\
\text { - Fortalecer, reorientar y/o ajus- } \\
\text { tar el proceso de formación, en } \\
\text { cuanto a materiales, estrategias, } \\
\text { contenidos, etc. } \\
\text { - Realimentar el proceso de for- } \\
\text { mación. }\end{array}$ & $\begin{array}{l}\text { - Permite identificar el grado de } \\
\text { dominio alcanzado por los estu- } \\
\text { diantes en cuanto a las compe- } \\
\text { tencias prescritas en el módulo } \\
\text { de aprendizaje; con objeto de } \\
\text { generar situaciones de enseñan- } \\
\text { za y aprendizaje que posibiliten el } \\
\text { logro de los resultados de apren- } \\
\text { dizaje aún no desarrollados. } \\
\text { - Permite tomar decisiones en } \\
\text { cuanto a su nivel de dominio de } \\
\text { la competencia: competente o } \\
\text { aún no competente, con el fin de } \\
\text { acreditar el módulo de aprendiza- } \\
\text { je, y en su caso orientarlos hacia } \\
\text { el proceso de evaluación y certifi- } \\
\text { cación de la competencia laboral. }\end{array}$ \\
\hline
\end{tabular}

\begin{tabular}{|c|c|c|}
\hline \multicolumn{3}{|c|}{ ¿Qué evaluar? } \\
\hline Diagnóstica & Formativa & Sumativa \\
\hline $\begin{array}{l}\text { - Conocimientos previos de los } \\
\text { que parten los estudiantes pa- } \\
\text { ra enfrentar los resultados de } \\
\text { aprendizajes propuestos en el } \\
\text { módulo de aprendizaje. } \\
\text { - Conocimientos que se tienen } \\
\text { sobre los resultados de aprendi- } \\
\text { zaje propuestos en el módulo de } \\
\text { aprendizaje. }\end{array}$ & $\begin{array}{l}\text { - Factores y elementos que inter- } \\
\text { vienen en el proceso de apren- } \\
\text { dizaje de la competencia en los } \\
\text { estudiantes. } \\
\text { - Avances del proceso de forma- } \\
\text { ción, a través de las evidencias } \\
\text { obtenidas hasta el momento. }\end{array}$ & $\begin{array}{l}\text { - Grado de dominio de la compe- } \\
\text { tencia propuesta en el módulo } \\
\text { de aprendizaje. } \\
\text { - Evidencias obtenidas y organi- } \\
\text { zadas en el portafolio de eviden- } \\
\text { cias. }\end{array}$ \\
\hline
\end{tabular}

\begin{tabular}{|l|l|l|}
\hline \multicolumn{2}{|c|}{ ¿Cuándo evaluar? } \\
\hline \multicolumn{1}{|c|}{ Diagnóstica } & \multicolumn{1}{|c|}{ Formativa } & \multicolumn{1}{c|}{ Sumativa } \\
\hline - Previo al inicio de un conjunto or- & - Durante todo el proceso de for- \\
ganizado de competencias: & mación. & Se realiza: \\
- Un módulo de aprendizaje. & & $\begin{array}{l}\text { Al final de cada unidad didáctica. } \\
\text { zaje. }\end{array}$ \\
- Una unidad didáctica. & & \\
\hline
\end{tabular}

La acreditación en la Capacitación se logra en la medida que el estudiante muestra, a través del proceso de evaluación, que es capaz de realizar las competencias establecidas en los resultados de aprendizaje de cada módulo, ya que no se puede emitir un juicio que valore al estudiante como suficiente o medianamente competente para realizar determinada función productiva.

En este sentido el proceso de formación es un espacio que contribuye a generar situaciones de enseñanza y aprendizaje que posibiliten realizar las 
funciones laborales correspondientes a un desempeño o la aplicación de una práctica. En cuanto a aquellos elementos y evidencias de la competencia que no se logren por los estudiantes en el periodo formal de aprendizaje - semestre escolar - serán evaluados particularmente en la llamada evaluación de recuperación.

De los aspectos desarrollados se desprenden distintos ejes y/o aspectos a tener en cuenta para hacer una propuesta de diseño:

\section{Estructura curricular:}

a) Conservación de la formación propedéutica y ampliación de las posibilidades de incorporación al sector productivo.

b) Atención de necesidades sociales de formación para personal de nivel "auxiliar".

c) Desarrollo de competencias laborales que permitan un desempeño eficaz en los capacitados.

d) Flexibilización del diseño curricular a través de la incorporación de los módulos de aprendizaje.

e) Diseño de la propuesta de actualización desde el campo referido a la "organización y procesamiento de la información".

f) Delimitación del nivel de amplitud del contenido en función de la duración de la capacitación.

g) Precisión del nombre de la capacitación y de las asignaturas con relación a las competencias esperadas y al nivel educativo que les corresponde, con el fin de evitar confusión en el perfil de egreso.

h) Selección de contenidos de lectura de acuerdo con las funciones básicas que lleva a cabo el personal "auxiliar" en las Unidades de Información.

i) Explicitación del campo de trabajo y de las salidas ocupacionales.

\section{Consideraciones para la operación:}

j) Incorporación de la tecnología y manejo de paquetes de aplicación específica del área; es decir, las herramientas indispensables para optimizar los procesos bibliotecarios.

Vinculación con el sector productivo:

k) Fortalecimiento de la vinculación con el sector productivo a través de las prácticas de campo. Obligatoriedad de las prácticas de campo.

Evaluación y certificación de la competencia laboral.

1) En tanto que actualmente no existen normas técnicas de competencia 
laboral de esta área emitidas por el CONOCER, sino de carácter institucional, se buscará el reconocimiento del desempeño de los estudiantes por parte de distintos empleadores.

Formación de personal académico.

m) Formación docente con el enfoque de competencia laboral del personal académico.

\section{c) Propuesta de actualización con el enfoque de competencia laboral}

Dado que se trata de una capacitación para prestar los servicios bibliotecarios y de información se propone que se llame: Capacitación en Servicios Bibliotecarios y de Información, cuya finalidad será:

Capacitar a los estudiantes para apoyar el desarrollo de sus competencias en la organización, registro y conservación del acervo que proporcionan los servicios bibliotecarios y de información, de manera manual y automatizada, y en la realización de las actividades y procedimientos de carácter técnico que les permitan el desempeño con calidad de las funciones laborales como parte del fortalecimiento de su formación propedéutica.

\section{Perfil del egresado:}

El alumno deberá ser capaz de desempeñarse en las funciones laborales para apoyar en los procesos de adquisición y registro de documentos, el mantenimiento de colecciones y catálogos, la utilización de instrumentos de búsqueda y recuperación de información, la prestación de servicios a usuarios, así como, en la difusión de los servicios bibliotecarios y de información en las diferentes bibliotecas y Unidades de Información

Como todo perfil incluye el conjunto de conocimientos, habilidades y actitudes que articulados sustentan el desarrollo de las competencias propuestas en la Capacitación en Servicios Bibliotecarios y de Información:

El alumno deberá tener conocimientos sobre:

- El proceso documental, las bibliotecas y las Unidades de información.

- El tipo de documentos.

- Los procesos de selección y adquisición.

- Los procesos físico manual y automatizado.

- La organización de catálogos.

- Los temas en la organización de catálogos. 
- La organización temática.

- La organización alfabética por título.

- El concepto y función de los inventarios.

- Los sistemas de búsqueda y recuperación de información.

- El manejo de programas de automatización de aplicación específica: SIABUC, Aleph (demo), Logicat (demo).

- Las áreas catalográficas de los documentos.

- La captura, la signatura topográfica y temas.

- Las bases de datos: Librunam, Tesiunam, Seriunam.

- La Internet como recurso de acceso a sistemas de búsqueda y recuperación de información confiable.

- Los medios para orientar al usuario en cuanto a los recursos documentales y los servicios bibliotecarios y de información.

- Las características que distinguen a las diferentes Unidades de Información:

- La filosofía del servicio que se ofrece en las bibliotecas.

- El préstamo de libros, las obras de consulta, las publicaciones periódicas y otros documentos (audiovisuales, cartográficos, etcétera).

- Los servicios de extensión bibliotecaria y de información en las bibliotecas públicas.

- El manejo de programas de aplicación específica: SIABUC, Aleph (demo).

- La difusión de los servicios bibliotecarios y de información.

- El control de préstamo manual y automatizado.

- El proceso de consulta de usuarios.

- Lasistematización de la cantidad de usuarios atendidos y los documentos prestados.

- La conservación de documentos.

- Los agentes que afectan a los documentos.

- Los factores que dañan el acervo impreso y digital.

- La identificación de documentos para reparación.

- La preparación de documentos para reparación.

Habilidades ${ }^{1}$ para:

- Realizar procedimientos de adquisición de documentos.

- Procesar físicamente libros, revistas y otras colecciones.

- Manejar catálogos de documentos.

- Incorporar físicamente libros revistas y otras colecciones al acervo.

1 Los elementos de las competencias se recuperan como las habilidades a desarrollar. 
- Ordenar físicamente el acervo.

- Auxiliar en el levantamiento de inventarios.

- Apoyar en la sistematización del proceso de adquisición de documentos.

- Apoyar en la sistematización de los procesos técnicos de documentos.

- Recuperar información de las bases de datos.

- Recuperar información en Internet.

- Orientar a los usuarios en el uso de los servicios bibliotecarios.

- Realizar préstamos de documentos.

- Apoyar la promoción y difusión de los servicios bibliotecarios.

- Manejar obras de consulta general.

- Controlar el acceso y salida del usuario.

- Tabular datos estadísticos de los servicios bibliotecarios.

- Mantener el acervo.

- Detectar documentos dañados.

- Reparar documentos que tienen daños menores.

- Recibir documentos que se enviaron a encuadernación.

Actitudes:

- Orden.

- Responsabilidad.

- Limpieza.

- Amabilidad.

- Tolerancia.

La Capacitación en Servicios Bibliotecarios y de Información organiza los contenidos para dar cuenta de la finalidad planteada, en módulos de aprendizaje que son considerados como unidades en sí mismas que contemplan, teórica y prácticamente, la totalidad del proceso para desarrollar cada una de las competencias propuestas.

En este sentido, la capacitación, propone el aprendizaje de cuatro competencias de carácter institucional-elaboradas por expertos en el área y con validez exclusiva para el Colegio de Bachilleres. ${ }^{2}$ Para cada una de las competencias que habrán de desarrollarse se establece un módulo de aprendizaje. Cabe señalar que como parte de las capacitaciones que ofrece el Colegio se imparte en el tercer semestre el módulo de aprendizaje común "Formación Laboral", como se observa en la siguiente tabla:

2 Las competencias que se proponen para la capitación tienen un carácter institucional, ya que en este momento el Consejo de Normalización y Certificación de Competencia Laboral no ha elaborada ninguna competencia para la subárea de biblioteconomía. 
Tabla No. 2. Módulos de aprendizaje para la Capacitación en Servicios Bibliotecarios y de Información

\begin{tabular}{|c|l|c|c|c|}
\hline Sem & \multicolumn{1}{|c|}{ Módulos } & $\begin{array}{c}\text { Horas/ } \\
\text { Semana }\end{array}$ & $\begin{array}{c}\text { Horas } \\
\text { Semestre }\end{array}$ & Créditos \\
\hline 3 & Módulo común: Formación Laboral & 4 & 64 & 8 \\
\hline & $\begin{array}{l}\text { Organizar recursos de información } \\
\text { Primera parte }\end{array}$ & 3 & 48 & 6 \\
\hline 4 & $\begin{array}{l}\text { - Organizar recursos de información } \\
\text { Segunda parte } \\
\text { - Sistematizar y recuperar informa- } \\
\text { ción }\end{array}$ & 4 & 64 & 8 \\
\hline 5 & $\begin{array}{l}\text { Proporcionar servicios a usuarios } \\
\text { - Conservar documentos }\end{array}$ & 7 & 78 & 6 \\
\hline TOT & & 7 & 444 & 14 \\
\hline
\end{tabular}

Debido a la amplitud de contenidos del primer módulo, Organizar recursos de información, éste se divide en dos partes con el fin de realizar las actividades de enseñanza y aprendizaje pertinentes y suficientes.

Las competencias a desarrollar en cada módulo son las siguientes:

- Organizar recursos de información.

Intención:

El alumno será competente para:

Organizar cualquier tipo de recurso informativo en las distintas Unidades de Información a través de situaciones cotidianas del ámbito bibliotecario con el fin de generar las condiciones necesarias para la búsqueda y recuperación de información.

- Sistematizar y recuperar información

Intención:

El alumno será competente para:

Aplicar procesos que agilicen la búsqueda y recuperación de información haciendo uso de los diversos medios y a través de situaciones cotidianos en el ámbito bibliotecario, para así proporcionar un servicio oportuno, pertinente y eficaz para el usuario.

\section{- Proporcionar servicios a usuarios}

Intención:

El alumno será competente para:

Realizar los servicios de atención al público y apoyar la difusión de los servicios que ofrecen las distintas Unidades de Información, a través de situaciones cotidianas del ámbito bibliotecario, para satisfacer las necesidades de información de los usuarios. 
- Conservar documentos.

Intención:

El alumno será competente para:

Preservar los documentos que conforman las diferentes colecciones de las Unidades de información, a través de situaciones cotidianas del ámbito bibliotecario con el objeto de mantener la colección en condiciones óptimas para brindar el servicio en las distintas Unidades de Información.

La Capacitación en Servicios Bibliotecarios y de Información esta diseñada de tal manera que el egresado podrá desempeñarse realizando actividades y procedimientos de orden técnico que sirvan de apoyo a la organización y registro de documentos, así como en la prestación de servicios en unidades de información y bibliotecas públicas, escolares, infantiles, universitarias, especializadas y nacionales; en centros de documentación e información, así como en librerías y empresas editoriales.

Al terminar cada módulo de aprendizaje los egresados de la capacitación serán competentes para realizar diversas funciones laborales que tienen relación con los diversos puestos de trabajo en que pueden desempeñarse. En las siguientes tablas se detalla la relación entre las funciones laborales correspondientes al primer módulo y los puestos asociados a la capacitación.

I. Módulo: Organizar Recursos de Información

\begin{tabular}{|c|c|c|c|c|c|c|c|}
\hline \multirow[b]{2}{*}{ Funciones laborales que desempeñan } & \multicolumn{7}{|c|}{$\begin{array}{c}\text { Puestos de trabajo que } \\
\text { desempeñan los egresados de } \\
\text { la capacitación }\end{array}$} \\
\hline & 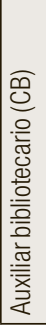 & 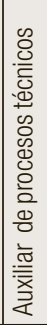 & 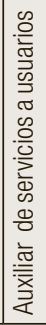 & 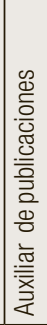 & 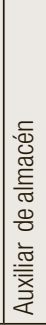 & 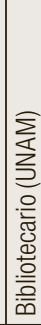 & 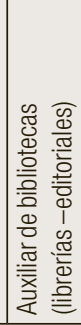 \\
\hline $\begin{array}{l}\text { 1. Realizar procedimientos de adquisición de } \\
\text { documentos }\end{array}$ & $x$ & & & & $x$ & & \\
\hline $\begin{array}{l}\text { 2. Procesar físicamente libros, revistas y } \\
\text { otras colecciones }\end{array}$ & $x$ & $X$ & & & $x$ & $x$ & \\
\hline 3. Manejar catálogos de documentos & $X$ & $X$ & & & & $X$ & \\
\hline $\begin{array}{l}\text { 4. Incorporar físicamente libros revistas y } \\
\text { otras colecciones al acervo }\end{array}$ & $x$ & & $x$ & & & $x$ & \\
\hline 5. Ordenar físicamente el acervo & $x$ & & $X$ & $\mathrm{X}$ & & & $x$ \\
\hline 6. Auxiliar en el levantamiento de inventarios & $x$ & & & $\mathrm{X}$ & $x$ & $x$ & \\
\hline
\end{tabular}


La forma de evaluar para determinar si se ha logrado la competencia se muestra a través de tres evidencias, una de producto y dos de desempeño. Gráficamente, la relación entre los diversos elementos del plan se muestra en la siguiente figura.

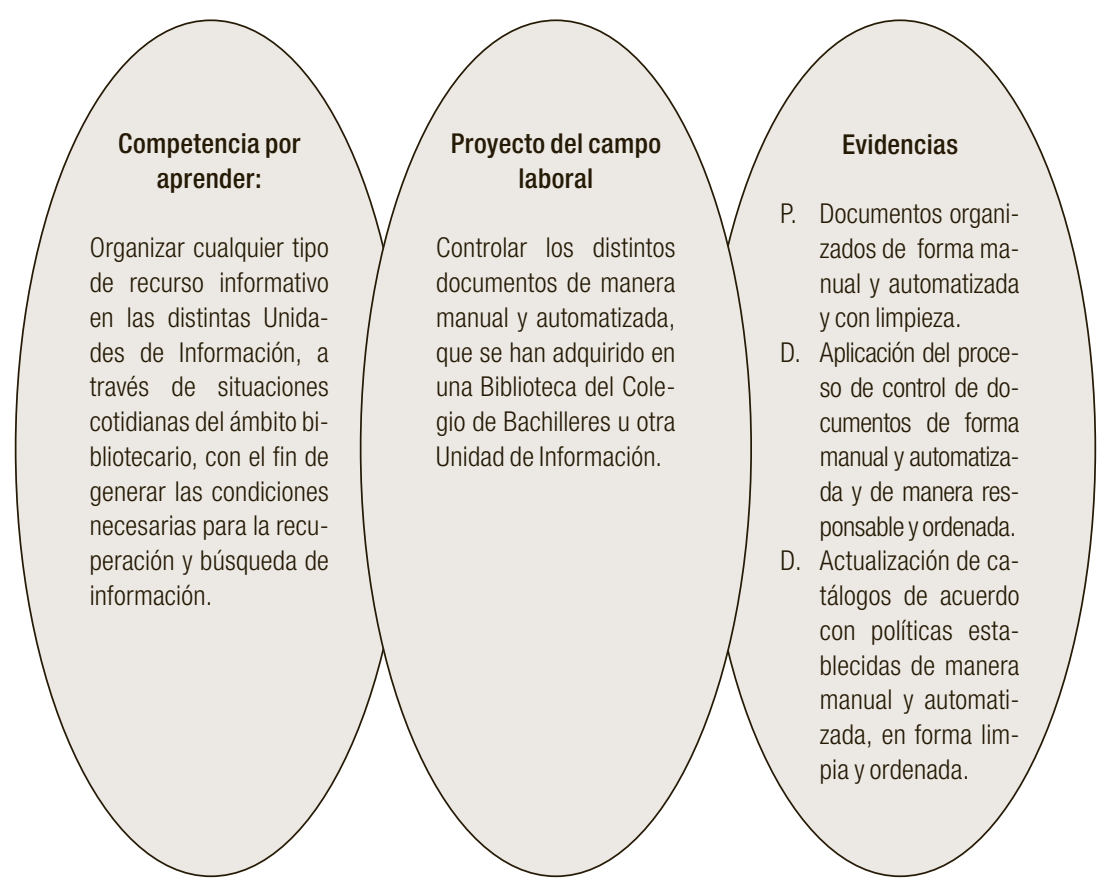

\section{Conclusiones}

El modelo educativo que tiene como base el enfoque de competencias que prepara a los estudiantes en el "saber hacer" de forma integral, es el adecuado para la capacitación en servicios bibliotecarios y de información, y se basa en el conocimiento teórico específico que le permite al alumno comprender lo que está haciendo y para qué lo está haciendo.

Por otra parte el enfoque aplicado a la capacitación está vinculado con el modelo educativo del Colegio de Bachilleres y llevará a la institución a la capacitación de calidad necesaria para la prestación adecuada de los servicios bibliotecarios y de información, ya que se contará con los conocimientos sobre los procesos que se llevan a cabo para la organización, conservación, sistematización y recuperación de la información, que día a día van creciendo a un ritmo acelerado. 
Este conocimiento que le es útil al estudiante para su incorporación al trabajo, cumple también con otra finalidad básica para el estudiante: su desarrollo y crecimiento académico, ya que podrá aplicar las competencias que desarrolle para la organización y recuperación de la información para su beneficio y en toda su actividad escolar, lo que repercutirá en un mayor aprovechamiento académico.

Sin embargo, es necesario considerar que un plan de estudios congruente y fundamentado no será exitoso si las autoridades no se comprometen con el enfoque y brindan la infraestructura física, tecnológica y académica adecuada para llevarlo a cabo. Un plan que se basa en la práctica de diversas actividades debe contar con laboratorios, computadoras, y los materiales bibliográficos que faciliten el desarrollo de las competencias.

Por otra parte es fundamental la formación docente en este enfoque. Dado que es relativamente nueva la aplicación de competencias en la educación mexicana, se hace necesario formar a los profesores para que apliquen el enfoque con todo lo que éste implica: el diseño del programa, su seguimiento, el diseño de las estrategias de enseñanza, las formas de evaluación, etcétera. Lo anterior implica que los docentes posean un dominio absoluto del enfoque y un compromiso real con la institución educativa con los alumnos y con su propio trabajo docente.

Finalmente y dado que el estudiante es el eje central del enfoque de competencias, es imprescindible que también conozca y se comprometa con el mismo para que, fuera del plan de estudios y de la guía del profesor, aprenda a aprender durante toda la vida, y mejore cada día sus actividades y su persona.

\section{Bibliografía}

Acevedo Caicedo, Myriam y Gloria García Oliveros (2000), "La evaluación de las competencias en matemáticas y el currículo: un problema de coherencia y consistencia”, en Bogoya Maldonado, Daniel,... [et al], Competencias y proyecto pedagógico, Colombia: Universidad Nacional de Colombia.

Agenda personal 2002, México : Colegio de Bachilleres.

Argudin, Yolanda (2005), Educación basada en competencias : nociones $y$ antecedentes, México : Trillas.

Barrón Tirado, Concepción (2000), "La educación basada en competencias en el marco de los procesos de globalización”, pp. 17-45, en Formación en competencias y certificación profesional, México, UNAM, Centro de Estudios sobre la Universidad. 
Cárdenas Salgado, Fidel Antonio y Fernando Sarmiento Parra (2000), "Desarrollo y evaluación de competencias en ciencias", en Bogoya Maldonado, Daniel, et al, Competencias y proyecto pedagógico, Colombia: Universidad Nacional de Colombia.

Decreto de Creación y Estatuto General (1975), México, Colegio de Bachilleres.

Escalona Orcao, Ana Isabel y Blanca Loscertales Palomar (2005), Actividades para la enseñanza y el aprendizaje de competencias genéricas en el marco del Espacio Europeo de Educación Superior, Zaragoza, Prensas Universitarias de Zaragoza.

Fernández de Zamora, Rosa María (1999), Mapa bibliotecario de la Ciudad de México, México, Universidad Nacional Autónoma de México, CUIB.

Formación en competencias y certificación profesional (2000), María de los Angeles Valle Flores, coord. México, Universidad Nacional Autónoma de México, CESU.

Gonczi, Andrew (1997), "Problemas asociados con la implantación de la educación basada en la competencia: de lo atomístico a lo holístico", en Formación basada en competencia laboral, Montevideo, Uruguay: CONOCER, CINTERFOR-OIT.

Hernández Pina, Fuensanta... [et al.] (2005), Aprendizaje, competencias y rendimiento en educación superior, Madrid, Muralla.

Instituto Nacional de Estadística, Geografía e Informática (2003) Bibliotecas por tipo, en http://www.inegi.gob.mx/est/contenidos/espanol/rutinas/ept.asp? $\mathrm{t}=$ mcul03 \&c=3130, consultado el 7 de junio de 2007.

Modelo educativo (1998), México, Colegio de Bachilleres.

Orientaciones para la evaluación del aprendizaje (1997), México, Colegio de Bachilleres, Dirección de Planeación Académica.

Orientaciones para la evaluación del aprendizaje: guía didáctica para el profesor (1996), México, Colegio de Bachilleres.

Plan de actualización de la Capacitación en Biblioteconomía (1993), México, Colegio de Bachilleres, Dirección de Planeación Académica, Centro de Capacitación para el Trabajo.

¿Qué es la evaluación de la competencia laboral?, México: CONOCER, [s.a.] [Folleto informativo].

Rendón Montenegro, Ignacio (2003), Aprendizaje y desarrollo de competencias, Bogotá, Cooperativa Editorial Magisterio.

Ríos Ortega, Jaime, "Modelos en conflicto en la formación del bibliotecólogo mexicano", [Mecanograma].

Schultz, Theodore W. (2000), "Capital humano", en El debate social en torno a la educación; enfoques predominantes. Comps. José Gómez Villanueva y Alfonso Hernández Guerrero, México, UNAM,ENEP Acatlán.

Tobón, Sergio... [et al.] (2006), Competencias, calidad y educación superior, Bogotá, Cooperativa Editorial Magisterio. 


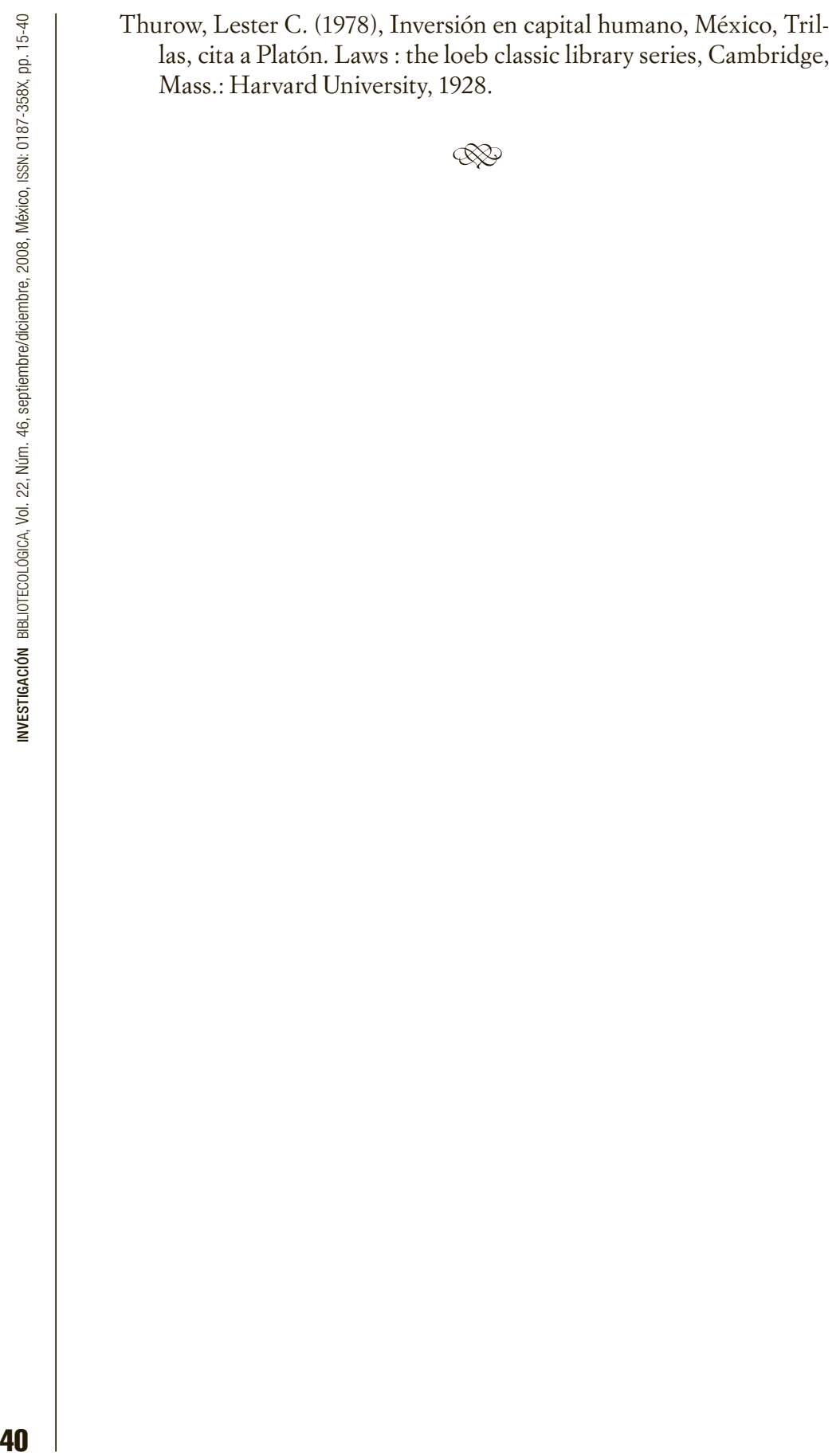

